\title{
RECONOCIMIENTOS ARQUEOLÓGICOS EN LA COSTA NORTE DEL PERÚ
}

\author{
David H. Kelley
}

La numeración de campo de yacimientos y los nombres tentativos para cultura dados en este informe están sujetos a cambios. Se espera que un sistema más regular de numeración de campo permanente sea dado, pero los nombres de culturas serán mayormente retenidos. Sólo se ha hecho un análisis muy preliminar de los materiales. Al presente, la evidencia de seriaciones y comparaciones con otros yacimientos peruanos situados al Sur no han dado resultados comparables y tampoco han sido suficientemente aclarados como para ser definitivos. Muy poca evidencia estratigráfica ha sido obtenible hasta ahora y este poco todavía no ha sido analizado. Sin embargo, un número de culturas arqueológicamente distintas han sido encontradas, muchas del ellas presentan, en más de un yacimiento sobre una distancia considerable, una diferencia mayormente cronológica que geográfica, aparentemente. Sobre esta base, varios periodos han sido distinguidos a pesar que su secuencia no ha sido todavía determinada. Muchos yacimientos no están incluidos en los periodos definidos, a causa de la poca cantidad de fragmentos de cerámica, o a lo esparcido de las características relativas a las culturas definidas. Lo último podría indicar, sea una continua ocupación por dos a más culturas definidas, una posición temporalmente intermedia entre culturas definidas, una persistencia de rasgos antiguos en un área geográficamente distinta, o una reocupación del sitio. La decisión entre estas posibilidades está sujeta a posteriores análisis.

Las siguientes culturas han sido definidas o reconocidas: Colán, Chusís, Sechura, Casita, Martín, San Pedro, Virrilá, Medanos, Cúchales y Matacaballo, para culturas mayormente en la zona costanera entre Boca Virrilá hasta la boca de Río Chira. Garabato, Cocos y Plateritos para otras áreas. Estas últimas podrán indudablemente ser extendidas en forma considerable cuando se hagan más análisis.

\section{Periodo San Pedro}

El yacimiento tipo es San Pedro Ocho, un conchal grande situado a corta distancia al norte del río Sechura, a la vista de la ciudad del mismo nombre. La cerámica generalmente contiene una sustancia metálica (ibroncita?). La forma más común (por impresión, no por recuento de fragmentos de cerámica) es una olla grande sin borde saliente, generalmente sin decoración pero ocasionalmente tiene un filete apliqué, cortado o entallado generalmente alrededor del lomo. Probablemente los fragmentos decorados forman considerablemente el 1\% del total. Excepto para los filetes, 
la decoración es muy escasa, cuando se la encuentra, es por lo general en tazones bajos, que es una forma rara. Un fragmento mostró una línea ancha, poco profunda, incisa. Otra, con una pasta gris poco común, provenía de un tazón achatado con borde hacia adentro, y mostraba una zona de "marca de peine" definida por 2 anchas líneas horizontales poco profundas. Siete fragmentos mostraban escisión, uno siendo una puntuación zonada, 4 siendo triángulos con líneas adentro ( 2 eran fragmentos de bordes con los triángulos pendientes de una línea justamente debajo del borde). Uno de estos fragmentos era de un tazón compuesto de anaranjado pulido, diferente mayormente de la cerámica del yacimiento, a pesar de tener un temperante similar. Dos fragmentos de cerámica mostraban dibujos escalonados y puntos en pintura blanca sobre una superficie natural de la vasija. Un fragmento estaba pintado en negativo o más o menos una decoración negativa con una hilera de puntos, entre dos líneas paralelas curvadas, la superficie habiendo sido en cierta forma oscurecida al fuego y la decoración mostrando encima un color ligeramente más claro. Obviamente el brochado interior es típico de la mayor parte de fragmentos.

Una trinchera de $2 \mathrm{~m} \times 2 \mathrm{~m}$, dio aparentemente un suelo estéril a más o menos $1 / 2 \mathrm{~m}$, y otros adicionales fueron puestos de costado hasta que tenía $2 \mathrm{~m}$ x $10 \mathrm{~m}$ y como $1 / 2 \mathrm{~m}$ de profundidad. Sólo un fragmento decorado fue hallado en este lugar. Otra trinchera de $2 \mathrm{~m}$ x $2 \mathrm{~m}$ no había encontrado suelo estéril a 2 $\mathrm{m}$ de profundidad, cuando la arena que caía impidió continuar excavando. Dos fragmentos decorados y 3 fragmentos de borde fueron encontrados en esta excavación.

Artefactos de piedra bruta, incluyendo rodados de cuarcita desgastados y varios tipos de artefactos de núcleo, fueron encontrados. Todos ellos de tipos encontrados en Colán (debajo) aunque los rodados de cuarcita (ilastre para redes?) eran comunes más que en Colán.

De varios cientos de montículos de conchas hacia el norte de este yacimiento, algunos de los otros probablemente pertenezcan a este periodo. Sin embargo la erosión completa de todos los fragmentos de cerámica de la superficie es una regla general en este sector casti- gado por el viento, y sólo raros fragmentos son encontrados sin hacer excavaciones. Algunos yacimientos mostraban cerámica que parecía en sus características de alfarería a San Pedro Norte, A17a. Fragmentos de bordes y características de la cerámica de este yacimiento definitivamente sugiere San Pedro 8, y hay, probablemente, un componente de este periodo allí, con los restos del Periodo Casita y probablemente algunos restos del Periodo Colán.

\section{Periodo Martín}

El yacimiento de San Pedro Norte A18 está en el acantilado encima de La Casita, a más o menos $1 \mathrm{~km}$ de San Pedro Norte A17, adyacente a 4-2 y cerca de La Casita 3. Desde que no hay un nombre local para este yacimiento, he llamado este periodo con el nombre de un amigo mío. El yacimiento muestra sólo fragmentos de cerámica esparcidos en un depósito delgado de arena o sobre la misma roca. La cerámica es delgada, (algunas veces muy delgada), a menudo de color gris a gris púrpura, bastante dura, y frecuentemente incisa. En algunos casos tiene un brochado vertical exterior y generalmente tiene un brochado interior obvio. La proporción de fragmentos de cuello a fragmentos incisos indican mucho menos incisión que el yacimiento 4-2 (ver Periodo Casita) pero la alfarería es a veces indistinguible. Sin embargo, desde que el yacimiento es continuo con el 4-2 no está claro si estos son fragmentos extraviados pertenecientes a los habitantes del yacimiento $4-2$ o bien si actualmente son los prototipos o descendientes de las formas 4-2. La más extraordinaria característica de la cerámica es un borde poco común, como el borde de una tetera, aparentemente designado para soportar una tapa. Dos de estas han sido encontradas. Un cuello de botella ha sido encontrado.

\section{Periodo Casita}

EL yacimiento tipo para este periodo es $4-2$, descubierto por Lanning y descrito por él como posiblemente correspondiente al Chavinoide terminal en el sur. Ambos, 4-2 y San Pedro 
Norte A17a, que tienen un componente de esta cultura, están en la inmediata vecindad de La Casita (1-2 km de distancia) que es la razón por la cual se le ha dado este nombre, si bien San Pedro Norte A18, La Casita 3 y San Pedro Norte A17b están también cerca. La alfarería es típicamente de una cerámica muy delgado gris con un interior blanquecino brochado. Los exteriores brochados no son raros, incluyendo brochado vertical. Hay un porcentaje bastante grande de cerámica decorada, mayormente incisa. La incisión es generalmente ancha y poco profunda, pero ocasionalmente profunda y delgada. Dos fragmentos mostraban incisión punzada y arrastrada. Filetes entallados en apliqué ocurren, así como incisiones lineales puntuadas. El modelo de incisiones en "chevron" es común. San Pedro Norte A17a es casi seguramente un yacimiento de más de un periodo de ocupación, pero las vasijas en forma de caras del yacimiento probablemente pertenezcan a este periodo, en base a la observación superficial de las similitudes en pasta y color, y un fragmento del último sitio muestra puntuaciones con juncos. Algunos fragmentos muestran líneas pintadas de muy bajo contraste. El yacimiento Chira 2, junto al Sur del río Chira, cerca de la Costa, es un pequeño depósito de conchas en un montículo, donde por todo encontramos sólo 23 fragmentos de cerámica. Dos de ellos estaban incisos con líneas anchas en una cerámica gris-marrón de temperante grueso y otros cuatro eran bastante delgados, con interiores blancos brochados, claramente la misma cerámica distintiva como en 4-2. Mientras que cuatro fragmentos no hacen un fundamento firme para sacar conclusiones, pueden sugerir que la cultura Casita represente una cultura bastante difundida y podría ser correctamente considerada como un periodo, más bien que una división geográfica. Varios artefactos de piedra sin tallar, parecidos a aquellos del periodo Colán en el yacimiento tipo han sido también encontrados en Chira 2.

\section{Periodo Sechura}

El yacimiento tipo está situado junto al sur del cementerio, a su vez al este de la actual ciudad de Sechura. Christensen considera la gente de Sechura como responsables de las cultu- ras Chusís y Sechura, que él tiende a igualar. Puesto que él ha aplicado el nombre de Chusís a la misma cultura que llamo yo Chusís, he optado por tomar el nombre de Sechura (que él usa primeramente como un nombre étnico más bien que como nombre de un yacimiento) para la cultura arqueológica encontrada en Sechura y en los yacimientos sin nombre de San Pedro Norte A20 y San Pedro Norte A21. Esto está sujeto a su aprobación. Los yacimientos de Sechura comparten con Chusís un tipo distintivo de líneas curvas muy anchas, pero la característica especial más distintiva que me ha guiado para formarlo como un periodo separado (quizás cubriendo en parte al periodo Chusís) es la presencia de varios bordes cortados y modelados muy especializados en vasijas y asas enrolladas aplanadas para vasijas, características que se repiten en SPN A20 y SPN A21 de Sechura en comparación con Chusís.

\section{Periodo Chusís}

Excavaciones en el yacimiento tipo de Chusís han sido hechas por Christensen, que ha señalado las afinidades Gallinazo generales de la cultura. Estas incluyen filetes horadados en apliqué, huacos en forma de cara discos de cerámica incisos en líneas punteadas perforadas o no, tipos especializados de husos y un tipo muy especializado de filetes grandes de cuello en apliqué y bordes dentados (impresos con el pulgar). Estos bordes muy distintivos me son conocidos sólo en Chusís y tienen su más cercano paralelo publicado en la cerámica Gallinazo, bien que también tienen algo en común con los bordes elaborados del Periodo Sechura. Christensen da cuenta de unos fragmentos raros con pintura negativa. Si bien notó las afinidades Gallinazo creía que estaba floreciendo todavía en los tiempos de la Conquista Española, y que probablemente comenzó no antes de 1000 A.D. Me parecería muy poco probable que hubiese durado hasta esa época tardía. La diferencia de opinión es primariamente, una cuestión sobre la interpretación de los dos niveles superiores de las excavaciones de Christensen y algunas semejanzas en la cerámica moderna de estas áreas a la cerámica Gallinazo. Este no es el lugar para intentar resolver un problema difícil y será suficiente notar que 
fuera de todas las culturas del departamento de Piura que conozco, con excepción de los últimos periodos, las afinidades de Chusís parecen ser más claras. En general, la alfarería es pesada y bien cocida, usualmente roja o negra. Las excavaciones de Christensen revelaron menos del $1.3 \%$ de cerámica decorada. También adquirí de pobladores de Chusís 3 vasijas en miniatura, un rasgo que se repite en Gallinazo. La naturaleza precisa de las afinidades Gallinazo quedan oscuras, pero los yacimientos Gallinazo son mucho más comunes que lo que se había creído anteriormente y se extienden más lejos al Norte. Tal es el yacimiento de Santo Domingo, en el valle de Moche, que corresponde cercanamente a Gallinazo, en todos sus detalles revela muchas similitudes a Chusís. Este sitio y Cerro Tambo Real me fueron llamados la atención por Richard Scheadel, y el yacimiento de Santo Domingo (bajo el nombre de Santa Isabel) también me fue llamado la atención por Sol Miller.

\section{Periodo Colán}

El yacimiento tipo es Colán Norte 1, y hay claramente una ocupación de este periodo en San Pedro Norte A17b. La alfarería más típica es una cerámica gruesa pulida, a menudo con un engobe blanco o rojo púrpura, raramente con un engobe anaranjado. La decoración más típica es un filete apliqué sencillo, que divide una zona de engobe rojo de una zona con engobe blanco. No se ha encontrado ningún fragmento de cerámica incisa en Colán Norte 1 y en San Pedro del Norte A17b. Tanto en Colán Norte 1 y en San Pedro Norte A17b encontramos fragmentos de vasijas con puntos blancos en una superficie interior pintada de rojo. Los filetes usados para separar o marcar la separación de la zona pintada de rojo, de la de blanco, se encuentran también en La Tortuga en alfarería muy similar y claramente pertenecen a una manera general, a este periodo. Un fragmento inciso de La Tortuga puede pertenecer a un diferente periodo de tiempo, o reflejar la rara presencia de incisión en el periodo Colán. Es posible que sólo indique una ligera diferencia temporal para el yacimiento como un todo del yacimiento de Colán.
No se ha analizado todavía un foso de prueba de Colán Norte 1, pero no he revelado la presencia de ninguna de las culturas reconocidas a la fecha, o desde entonces, si bien los análisis probablemente harán posible que el periodo Colán se desdoble en dos subdivisiones.

La diferencia mayor entre estos yacimientos y un rango que alinea Colán con el periodo San Pedro, es la gran frecuencia de artefactos en piedra bruta en Colán Norte 1. Estos son generalmente núcleos poco trabajados de uso muy incierto, cantos rodados bastante grandes, rotos de manera a dejar un borde cortante o para raspar, o cantos de cuarzo golpeados en los terminales.

Colán Norte1 es un grupo de más de 20 montículos de conchas (conchales) situados en los bordes de una antigua (pero geológicamente reciente) laguna y la subsistencia debe haber sido debida a la pesca, ya que parece haber habido un mayor énfasis en la pesca de conchas. San Pedro Norte está situado en la escapa de playa y las plataformas y casas son de caliza cortada. Tremendas cantidades de huesos de lobo de mar fueron notadas en un punto. El área es claramente no apropiada para la agricultura y la base de subsistencia debe haber sido la pesca y la caza de mamíferos marinos.

Afinadas de fuera parecen existir en las llamadas culturas "blanco sobre rojo", pero de ninguna manera estas son claras. La ausencia completa de incisiones de cualquier tipo o excisión, indican una marcada diferencia del periodo San Pedro (y por supuesto de todas las culturas arriba indicadas) pero los artefactos de piedra bruta parecen indicar una conexión cercana con el periodo San Pedro, que no se encuentra en las otras culturas.

\section{Periodo Virrilá}

El yacimiento típico es S6 un conchal en un acantilado cerca de la Costa, al Sur de Boca Virrilá. No se han encontrado fragmentos de cerámica decorados aquí y la forma típica es aparentemente una jarra de cuello alto con un labio saliente. Esta misma forma se repita en San Pedro Siete, donde es el tipo dominante en la escasa colección obtenida en superficie. 
Pasta, temperante y superficie son, por lo menos superficialmente, muy similares. También se encontró un pequeño número de bordes del periodo Médanos en San Pedro Siete.

\section{Periodo Médanos}

El yacimiento tipo es Médanos 2, pequeño yacimiento en el Norte de la carretera PiuraSechura (en el antiguo camino a La Tortuga). El yacimiento consiste mayormente de conchas y fragmentos de cerámica esparcidos en la superficie. El yacimiento no hubiera sido considerado representativo de un periodo excepto que los escasos fragmentos de cerámica encontrados correspondían cercanamente con la muestra poco mayor de San Pedro Cinco (que hubiera sido el yacimiento tipo, excepto que el nombre San Pedro ya había sido usado par ala cultura de San Pedro Ocho). San Pedro Cinco está probablemente a menos de $200 \mathrm{~m}$ de San Pedro Siete, y contenía unos cuantos bordes típicos de San Pedro Siete, junto con su propio borde distintivo, un cuello alto, grueso y derecho, con un labio redondo y engrosado.

Se encontró un fragmento decorado, un asa incisa. La incisión está mejor que aquella del periodo Chuchales (abajo), pero reminiscencias de las asas incisas representando manos en el siguiente periodo. La continuidad entre los periodos Médanos y Virrilá está sugerido por la presencia, en cantidades variables de ambos tipos de fragmentos en San Pedro 5 y San Pedro 7, tanto así como la similitud básica en características cerámicas, incluyendo el uso de jarras de cuello alto y recto, a pesar de que el labio está diferentemente tratado. Los datos al presente no son adecuados para siquiera sugerir cual de los dos periodos es más temprano, ni donde vienen con respecto a las culturas previamente descritas. Es mi impresión de que son probablemente inmediatamente anteriores a la cultura Cúchales, basado en la apariencia de la pasta, el montículo de conchas y el pequeño depósito de conchas como patrones de población, y la presencia de bordes ocasionales del tipo Médanos y Virrilá en algunos yacimientos de los periodos Cúchales o matacaballo (notablemente S2). Allí probablemente indican una ocupación continuada.

\section{Periodo Cúchales}

La palabra Chuchal es el término local para un montículo de conchas pero también es dado como nombre propio de ciertos montículos de conchas específicos. El nombre parecía particularmente apropiado, ya que grandes montículos de conchas son un rasgo típico de la cultura de este periodo en esta área. El yacimiento tipo de un grupo de cinco montículos llamados chuchales, algo al noreste del Balneario Leguía, cerca de donde el río Piura entra la mar. Estos son montículos de conchas que han sido también usados para entierros y han sido malamente huaqueados. La cerámica es típicamente paleteada, con una muy amplia gama de dibujos. Sin embargo, el moldeado a presión es también una técnica decorativa frecuente, particularmente en vasijas bajas de lados rectos con argollas anulares de base. Pequeñas asas incisas, en forma de manos, son rasgos notables y ampliamente difundidos de la cerámica, si bien no muy comunes en ningún yacimiento en particular. Un fragmento de cerámica de chuchales era un cuello de botella, con un triángulo pendiente punteado inciso en él, de una manera muy reminiscente de la cultura Teatino. Yacimientos del periodo Chuchales son frecuentes desde el valle de Chao al Norte. Tienden a estar concentrados a lo largo de la Costa, y aún cuando están tierra adentro, un rasgo típico es la presencia de grandes cantidades de conchas de mar. El porcentaje de cerámica "paleteada" en estos yacimientos parece decrecer tierra adentro, volviéndose bajo en muchos de los yacimientos contemporáneos del valle de Lambayeque, y también mucho menos importante, aparentemente (hasta donde llegan mis conocimientos actuales) en la zona alta de los ríos Chira y Piura. En el Chira, la técnica del "paleteado" es todavía importante en Chalacalá, pero aparentemente no más tierra adentro de allí. En el Piura, es sólo una cerámica sin importancia en yacimientos tales como Tambo Grande, que sin duda son mayormente contemporáneos con las culturas Chuchales y Matacaballos. Aparte del yacimiento epónimo de Chuchales, yacimientos típicos de este periodo en el área bajo consideración son Colán 1 (al Este de la Hacienda Sojo), S2, varios yacimientos en la quebrada Nonura, tales yacimientos entre San Pedro y La Casita, y varios otros. Estos yacimientos cu- 
bren la zona de Costa de tal manera de que yo no puedo imaginar que la gente que habitaba contemporáneamente en el área hayan podido vivir sin compartir estas características.

Christensen ha asociado esta cultura con los tallanes de los cronistas (o más bien, esta cultura y Matacaballo), pero dudo que la cerámica tenga una asociación tan estrecha con un grupo étnico particular, aún en yacimientos de tipo Chuchales relativamente. Me parece poco probable, por ejemplo, que hubieron Tallanes en Sincape, al sur del San Pedro de Lloc, y por otro lado, muy probable que los sechuranos que vivieron en Chuchales, no eran Tallanes. Por esta razón, un nombre puramente arqueológico como el de chuchales, me parece preferible a un nombre de carácter étnico. Scheadel me informa que considera este periodo ser el equivalente cronológico de las culturas de la costa norte que él llama Tiahuanaco II, y hasta donde comprende el uso de su término estoy en pleno acuerdo.

\section{Periodo Matacaballo}

El yacimiento tipo es S11, un montículo de conchas situado al Este y un poco al Norte del poblado pesquero de Matacaballo. Las características del periodo Matacaballo son un aumento considerable en el uso del "paleteado" y en el abandono virtual de otra forma de decoración, acompañada por una gran disminución en los tipos de "paleteado" que virtualmente se restringieron a diferentes variedades de "achurados cruzados" (suedo-fiber-impressed).

Un número considerable de yacimientos Matacaballo, vidrios rotos de botella, excremento de cabra, clavos de hierro y otras indicaciones de persistencia a través del periodo colonial o posterior, pero mucho carecen de estas características. Ninguna clasificación global de los yacimientos de cerámica "paleteada" ha sido hecha. Soy de la opinión de que ciertos rasgos a la larga pueden permitir la división del periodo durante el cual se usó el macado con la técnica del "paleteado" en una serie de divisiones más pequeñas, aún con una base de seriaciones, sin evidencia estratigráfica. En este caso, es posible de que algunos de los yacimientos que yo voy a intentar clasificar como pertenecientes al periodo Mactacaballo, en base al número limitado de dibujos en "paleteado" y la ausencia de otras técnicas, puede actualmente pertenecer a un periodo anterior al de Chuchales. Por otro lado, varios yacimientos del periodo Chuchales indudablemente continúan siendo ocupados dentro de los tiempo de Matacaballo, pero la presencia de rasgos más elaborados oscurece la presencia de la cultura matacaballo, desde que se distingue principalmente por la ausencia de estos rasgos. No he hecho ninguna preferencia a los periodos Chimú o Inca, ya que el nombre que he puesto al periodo se refiere solamente al periodo durante el cual una cultura arqueológica particular que podría ser llamada Chimú o Inca está ocupando la zona en todos los yacimientos que he visitado, si bien fragmentos originales Chimú hasta Inca son encontrados en los yacimientos de Chuchales y Matacaballo. Es mi impresión que los fragmentos de cerámica negra convencionalmente llamados Chimú o Tallán son hallados en yacimientos que de otro modo serían clasificados como yacimientos Matacaballo y es posible que esta impresión sea en parte un artefacto de clasificación, en aquel uso extensivo de cualquier técnica diferente del "paleteado" me haría dudar sobre la clasificación del Matacaballo. En cualquier caso, a pesar de su representación extensiva en colecciones, la Hda. El Sojo es el único yacimiento que he visto en el que cerámica negra o gris a "presión" o modelada represente más de una muy pequeña fracción de la cerámica y aún allí no eran dominantes entre los fragmentos que he recolectado.

En la secuencia de periodos arriba mencionada, el llamado periodo Floreciente parece faltar completamente, a menos que los periodos muy poco impresionantes de Virrilá y Médanos sean ubicados en este lapso y es posible que los periodos experimentales duraron hasta el comienzo del periodo Chuchales. Sin embargo, parece más probable que los yacimientos de este periodo todavía quedan por descubrir en el valle de Piura. Algunos fragmentos de cerámica de Narigualá en una de las secuencias descubiertas y Tacalá no solo tiene materiales de la cultura Chuchales y fragmentos de cerámica con parecidos a las culturas de Chusís y Sechura, sino también muestra algunos rasgos 
únicos, que posiblemente pertenecen a los periodos medios.

En otras áreas, donde se han hecho estudios mucho menos extensivos, se han reconocido algunas culturas locales. Entre estas las siguientes perecen de mayor importancia:

\section{Cultura Garabato}

El yacimiento tipo es la Hda. Garabato en el flanco norte del río Chira, cerca de Sullana. Varias vasijas mezcladas Mochica-Tiahuanaco han prevenido de este yacimiento y algunas vasijas únicas que probablemente pertenecen a los periodos florecientes de Piura. Bordes ocasionales se parecen a aquellos del periodo Virrilá y hay clara ocupación Chuchales del yacimiento, así como una relativamente gran cantidad porcentual de cerámica negra "Chimú”. Sin excavaciones estratigráficas, será imposible definir propiamente la cultura claro de que una debería ser reconocida, aún su no está claramente definida.

\section{Cultura Cocos}

El yacimiento tipo es C-3, un yacimiento constituido por un cementerio y un lugar de habitación en el área ahora inundada por la represa de Los Cocos. Esta está un tanto tierra adentro de la costa, pero la amenaza de destrucción inmediatamente sugirió el valor de algunas excavaciones para obtener la mayor cantidad de datos posibles. Algo como 18 entierros fueron removidos. El número preciso puede ser probablemente determinado por el estudio cuidadoso de las anotaciones de campo, sumado al examen del material óseo. Un entierro intrusito estaba flexionado y sentado, pero todos los otros estaban extendidos en varias posiciones diferentes, a menudo con partes de unos cortados por varios otros. Los restos de las tumbas incluían una o más vasijas de cerámica y generalmente una pulsera de cuentas. Un entierro tenía un depilador de cobre y varios discos de cobre de pendientes, que no estaban claramente asociados con ningún entierro, fueron encontrados durante la excavación. Con una excepción, la cerámica era bastante uniforme, pequeñas ollas de cocina de pasta color naranja con un temperante metálico y dos pequeñas asas en lazo en un collar bajo. La excepción era una jarra gris con un asa de banda incisa. Esta era la única cerámica decorada encontrada en el yacimiento o en la región, excepto para materiales de un cemente "Inca-Chimú", vasijas silbadoras dobles, etc. Sin mayores análisis y comparaciones, poco puede decirse sobre esta cerámica decididamente no distintiva.

\section{Cultura Culebras}

Esta cultura ha sido definida por el trabajo de Edward Lanning hecho para Frederic Engel. Hice un pozo de prueba en un yacimiento grande que me fue indicado por Lanning, catalogado como Huarmey Norte 1. Las muestras, aunque pequeñas, claramente mostraban la misma cultura precerámica de Culebras. Se encontró maní, algodón, lúcuma y otras evidencias de plantas domesticadas. El yacimiento se extiende por casi un kilómetro en el lado Este de una cadena de cerros que lo separan del mar. Va hacia abajo hasta lo que ahora es lecho lacustre seco. La presencia de productos agrícolas y la gran extensión de restos de conchas sugieren la posibilidad de que la laguna no estaba seca en el tiempo de ocupación o que el clima era más húmedo. No se encontró cerámica en la superficie o en el foso de prueba, y unido por la similitud a los restos de Culebras, inmediatamente al Norte parece no haber razón para decir que este yacimiento es (localmente) precerámico.

Aparte de estos yacimientos en que se han hecho trabajos, o que han formado parte del reconocimiento extensivo de la Costa Norte Lejana, se gastó una cantidad considerable de tiempo visitando yacimientos a través de toda la Costa Norte y se hicieron colecciones de superficie de casi todos los yacimientos visitados. Los siguientes yacimientos parecen de especial interés por una u otra razón:

Las Aldas.- Un centro ceremonial bastante grande o más o menos medio camino entre Culebras y Casma en la Costa, rodeado por zona de habitación grande. El sitio parece que 
estaba deshabitado desde tiempos precerámicos, a través del periodo de cerámica llana de Las Aldas hasta el periodo Chavinoide, Se deben realizar mayores trabajos con miras de verificar y extender la secuencia estratigráfica y determinar definitivamente si las estructuras ceremoniales son mayormente del periodo Aldas, que ahora parece probable, o si bien, son chavinoides. El uso de superficie de piedra, pilares de basalto y yeso, hacen de este yacimiento temprano un sitio de interés inusitado. Un mapa adecuado del yacimiento es también muy necesario, puesto que el publicado por Engel es decididamente engañoso.

Santo Domingo.- Los extensos y profundos depósitos en este yacimiento, a pesar de estar situados en una ladera, sugieren secuencia estratigráfica y una sección dentro del periodo Gallinazo I, que está principalmente representado en el yacimiento, que está situado en el valle de Moche.

Pacatnamú. - Este extenso yacimiento al Norte de Pacasmayo ha producido la única colección de textiles mochicas conocidos y algunas decoraciones incisas muy curiosas. Los fragmentos de cerámica superficiales producen una mayoría de especimenes podrían encajar muy bien en cualquier yacimiento del periodo Chuchales. Exámenes estratigráficos aquí serían muy prometedores.

Cerro de Talambo.- Este yacimiento, un poco al Sur y Este del Guadalupe, en la carretera a Cajamarca, produjo fragmentos variables de estilo chavinoide, Mochica "paleteado" y colonial (probablemente). Parece ser un muy buen sitio para excavaciones estratigráficas, aunque no se han encontrado basurales prometedores.

Cabeza de León.- El yacimiento se extiende desde el borde hacia el Sur a lo largo de la ruta del antiguo camino Inca, al pie de una larga cadena de cerros. Hay probablemente por lo menos 200 construcciones de piedra grande casas, plataformas, etc. La cerámica en superficie es rara.

Purgatorio.- Las anotaciones de Scheadel duramente hace justicia a este yacimiento grande e importante, justo al Este de Túcume y al Norte de Lambayeque.
Huaca Grande del Túcume.- Este es un montículo piramidal bastante construido encima de un montículo de conchas más antiguo. Todos los fragmentos de cerámica de este último que se encuentran era de una cerámica de este último que se encontraron era de una cerámica sin decoración con bordes sencillos ligeramente redondeados. La preservación de material vegetal en el montículo de conchas es buena y un corte estratigráfico aquí es urgentemente necesitado.

Apurle.- Es el yacimiento más grande al Norte de Chan Chan y un poco más temprano. La publicación de los datos completos de Scheadel sobre este yacimiento serían un gran avance, pero excavaciones serían muy necesarias.

Batán Grande.- Excavaciones científicas de por lo menos algunos de los entierros aquí es muy necesaria. Los ricos ornamentos de oro encontrados están siendo ya sea fundidos o vendidos ilegalmente a coleccionistas privados.

Q. Nonura y Punta Avip.- Estas dos áreas en la zona del carro Illescas han producido ambas cuencos delgados de piedra fielmente asociados con entierros no cerámicos. A pesar de dos viajes a Q. Nonura, no pude localizar el supuesto terreno con entierros no cerámicos. Esto sería de mucho provecho si se pudiera llevar a cabo. El señor Kostritsky podría proporcionarnos una mayor información sobre la localización del yacimiento, con ayuda de una fotografía aérea de la zona.

El Estero.- Este yacimiento está en un área de acceso muy difícil, a pesar que el mapa indica una carretera nivelada, que puede haber sido real hace veinte años. He hecho 3 intentos para encontrarla y debe haber pasado muy cerca de ella. El Dr. George Petersen me informa que el yacimiento había sido virtualmente limpiado por Barrington Brown, pero parece que debería haber algo en el área de la extensión indicada por Barrington Brown. Se debe llevar una cantidad extra considerable de gasolina. El yacimiento es posiblemente el más antiguo conocido en el Perú, excepto algunos yacimientos de las alturas. Hasta material para fechado C-14 sería extremadamente importante. 
Narigualá.- Este yacimiento en el valle de Piura ha dado indudablemente una vasija Chavinoide, con una cerámica de engobe blanco, junto con muchos materiales posteriores. En uno de los yacimientos más grandes en el valle de Piura, un mapeo completo y alguna excavación sería muy útil a pesar de que no se obtendría una amplia estratigrafía.

Tambo Grande.- Una colección en posición de Carlos Schaefer, de la Hacienda Angostura, todo supuestamente de la inmediata vecindad de Tambo Grande, incluía un número de vasijas Mochica así como varios tipos desconocidos. Un examen completo de esta área es definitivamente indicado.

Garabato.- Excavaciones aquí deberían proveer datos estratigráficos valiosos -ver datos previos.

Chalacalá.- Este yacimiento grande en el Chira (más o menos 20 montículos) es reminiscente, en cierta manera, de Purgatorio. Parece estar cerca al límite entre la cerámica "paleteada" y el uso de cerámica sin adornos y debe proporcionar datos valiosos sobre secuencias relativas y cross datings.

Colán Norte 1.- La probabilidad de que mayores excavaciones aquí darán buenos frutos es grande, y que den información adecuada para subdividir el período Colán y quizás también a pesar de que yo no los he encontrado, de periodos más tempranos (no creo que la posibilidad de resultados útiles haciendo mayores excavaciones en San Pedro 8 sea alta).

Plateritos.- Ver previamente.

Uña del gato.- Este yacimiento en el río Zarumilla ha producido cerámica incisa y pintada muy interesante, algo reminiscente a San Pedro 8, pero con muchos rasgos tardíos, particularmente en la forma de las vasijas. Sin embargo, la cerámica que fue mostrada por el Dr. Petersen bien podría ayudar en el problema de las relaciones peruano-ecuatorianas.

Una lista completa de los yacimientos visitados y otra de recomendaciones, acompañarán mi informe definitivo. 